Revue d'histoire de l'Amérique française

REVUE D.HISTOIRE DE L'AMÉRIQUE FRANÇAISE

NISH, Cameron, compilateur. « Le Canada français, origine à

1763. Cotes 6-1 ; 6-2 ; 6-3 ; 7 / Codes 6-1 ; 6-2 ; 6-3 ; 7 ». Bibliographie pour servir à l'étude de l'histoire du Canada français, bulletin $n^{0} 4$ (déc. 1968). Centre d'étude du Québec, Sir George Williams University, Montréal.

\title{
H.-Paul Thibault
}

Volume 22, numéro 4, mars 1969

URI : https://id.erudit.org/iderudit/302842ar

DOI : https://doi.org/10.7202/302842ar

Aller au sommaire du numéro

Éditeur(s)

Institut d'histoire de l'Amérique française

ISSN

0035-2357 (imprimé)

1492-1383 (numérique)

Découvrir la revue

Citer ce compte rendu

Thibault, H.-P. (1969). Compte rendu de [NISH, Cameron, compilateur. " Le

Canada français, origine à 1763 . Cotes $6-1 ; 6-2 ; 6-3 ; 7$ / Codes 6-1 ;6-2 ;6-3; 7 ». Bibliographie pour servir à l'étude de l'histoire du Canada français, bulletin $\mathrm{n}^{0} 4$

(déc. 1968). Centre d'étude du Québec, Sir George Williams University,

Montréal.] Revue d'histoire de l'Amérique française, 22(4), 655-656.

https://doi.org/10.7202/302842ar d'utilisation que vous pouvez consulter en ligne. 
Nish, Cameron, compilateur. "Le Canada français, origine à 1763. Cotes 6-1; 6-2; 6-3; 7 / Codes 6-1; 6-2; 6-3; 7”. Bibliographie pour servir à l'étude de l'histoire du Canada français, bulletin $n^{\circ} 4$ (déc. 1968). Centre d'étude du Québec, Sir George Williams University, Montréal.

Le dernier bulletin du Centre d'Etude du Québec poursuit l'inventaire des documents disponibles au Centre de Recherche en Histoire Economique du Canada français.

Il comprend sous la cote 1 une cinquantaine de pages d'abréviations auxquelles il faut se référer continuellement. La seconde partie, cotée 6-1, reprend intégralement l'inventaire des documents originaux sur microfilm publié dans le bulletin $n^{\circ} 1$ et $y$ ajoute les bobines 31 à 48 qui proviennent des Archives publiques du Canada, des Archives départementales de la Gironde, des Archives départementales de la Charente-Maritime, des archives du Château de Ramesay et de la Société historique de Montréal, et de celles de la Bibliothèque Nationale du Québec. Suit une classification par noms propres (cote 6-2) et par sujets (6-3) des documents sur Xerox ou photocopiés en majorité aux Archives du Québec et aux Archives publiques du Canada. Enfin, la dernière partie (cote 7) complète l'inventaire des documents originaux imprimés du premier bulletin.

Une fois de plus, nous devons admettre que Cameron Nish accomplit une tâche gigantesque en montant de toutes pièces un fonds d'archives qui relègue au second plan nos propres Archives du Québec, du moins en ce qui a trait au régime français du XVIII ${ }^{\mathrm{e}}$ siècle.

L'inventaire qu'il nous en fait comporte cependant de nombreuses lacunes. Notons, sur le plan technique, l'absence totale de pagination, une impression verticale et trop souvent illisible, l'inexistence des signes de ponctuation et des signes grammaticaux usuels (apostrophe remplacé par un sigle incompréhensible, "ç" inexistant, etc.). Tout cela nous fait préférer de beaucoup la présentation du bulletin $n^{\circ} 1$.

La classification par noms propres et par sujets des documents sur Xerox ou photocopie est une amélioration par rapport aux bulletins précédents; mais l'auteur ne nous fournit toujours pas les critères de cette classification.

Nous demeurons sous l'impression que si cet ouvrage peut servir de quelque façon à l'étude entreprise par Cameron Nish, l'utilisation en est presque impossible pour ceux qui poursuivent des recherches différentes ou plus précises que la sienne. 
Nous n'avons rien contre l'emploi de l'ordinateur électronique en histoire, bien au contraire; mais pour qu'un tel outil produise un travail utile et utilisable, il faut d'abord une programmation cohérente et pratique, axé sur de multiples fins. Le présent bulletin ne reflète pas cette préoccupation.

Quoi qu'il en soit, espérons que les bulletins de Bibliographie pour servir à l'étude de l'histoire du Canada français continueront à s'améliorer et qu'ils atteindront dans un avenir prochain le stade de l'utilisation facile.

Parc historique national

H.-PaUl ThibaulT

Louisbourg, N.-E. 